\title{
A Statistical Model for Near-Synonym Choice
}

\author{
DIANA INKPEN \\ University of Ottawa
}

\begin{abstract}
We present an unsupervised statistical method for automatic choice of near-synonyms when the context is given. The method uses the Web as a corpus to compute scores based on mutual information. Our evaluation experiments show that this method performs better than two previous methods on the same task. We also describe experiments in using supervised learning for this task. We present an application to an intelligent thesaurus. This work is also useful in machine translation and natural language generation.
\end{abstract}

Categories and Subject Descriptors: I.2.7 [Artificial Intelligence]: Natural Language Processing-text analysis; I.2.6 [Artificial Intelligence]: Learning-induction; knowledge acquisition

General Terms: Algorithms, Languages, Performance

Additional Key Words and Phrases: Lexical choice, near-synonyms, semantic similarity, Web as a corpus, intelligent thesaurus

\section{INTRODUCTION}

When writing a text, a poorly chosen word can convey unwanted connotations, implications, or attitudes. Similarly, in machine translation and natural language generation systems, the choice among near-synonyms needs to be made carefully. By near-synonyms we mean words that have the same meaning, but differ in lexical nuances. For example, error, mistake, and blunder all mean a generic type of error, but blunder carries an implication of accident or ignorance. In addition to paying attention to lexical nuances, when choosing a word we need to make sure it fits well with the other words in a sentence. In this paper we investigate how the collocational properties of near-synonyms can help with choosing the best word in each context. This problem is difficult because the near-synonyms have senses that are very close to each other, and therefore they occur in similar contexts.

The work we present here is needed in two of our applications. The first one is an intelligent thesaurus. A writer can access a thesaurus to retrieve words that are similar to a given word, when there is a need to avoid repeating the same word, or when the word does not seem to be the best choice in the context. A standard thesaurus does not offer any explanation about the differences in nuances of meaning between the possible word choices. Moreover, a standard thesaurus tool does not attempt to order the choices to suit a particular writing context. Our intelligent thesaurus offers explanations and orders the choices using their

Author's address: School of Information Technology and Engineering, University of Ottawa, 800 King Edward, Ottawa, ON, Canada, K1N 6N5

Permission to make digital/hard copy of all or part of this material without fee for personal or classroom use provided that the copies are not made or distributed for profit or commercial advantage, the ACM copyright/server notice, the title of the publication, and its date appear, and notice is given that copying is by permission of the ACM, Inc. To copy otherwise, to republish, to post on servers, or to redistribute to lists requires prior specific permission and/or a fee.

(C) 2007 ACM 0000-0000/2007/0000-0001 $\$ 5.00$ 
Sentence: This could be improved by more detailed consideration of the processes of ......... propagation inherent in digitizing procedures.

Original near-synonym: error

Solution set: mistake, blooper, blunder, boner, contretemps, error, faux pas, goof, slip, solecism

Sentence: The day after this raid was the official start of operation strangle, an attempt to completely destroy the ......... lines of communication.

Original near-synonym: enemy

Solution set: opponent, adversary, antagonist, competitor, enemy, foe, rival

Fig. 1. Examples of sentences with a lexical gap, and candidate near-synonyms to fill the gap.

collocational properties relative to the writing context.

The second application is a natural language generation (NLG) system [Inkpen and Hirst 2003] that uses symbolic knowledge of near-synonym differences. This knowledge was acquired by applying information extraction techniques to entries in various dictionaries. We included a preliminary collocation module that reduces the risk of choosing a near-synonym that does not fit with the other words in a generated sentence (i.e., violates collocational constraints). The work presented in this paper allows for a more comprehensive near-synonym collocation module.

More specifically, the task we address in this paper is the selection of the best near-synonym that should be used in a particular context. The natural way to validate an algorithm for this task would be to ask human readers to evaluate the quality of the algorithm's output, but this kind of evaluation would be very laborious. Instead, we validate the algorithms by deleting selected words from sample sentences, to see whether the algorithms can restore the missing words. That is, we create a lexical gap and evaluate the ability of the algorithms to fill the lexical gap. Two examples are presented in Figure 1. All the near-synonyms of the original word, including the word itself, become the choices in the solution set (see the figure for two examples of solution sets). The task is to automatically fill the gap with the best choice in the particular context. We present a method of scoring the choices. The highest scoring near-synonym will be chosen. In order to evaluate how well our method works we consider that the only correct solution is the original word. This will cause our evaluation scores to underestimate the performance of our method, as more than one choice will sometimes be a perfect solution. Moreover, what we consider to be the best choice is the typical usage in the corpus, but it may vary from writer to writer. Nonetheless, it is a convenient way of producing test data in an automatic way. To verify how difficult the task is for humans, we perform experiments with human judges on a sample of the test data. The statistical scoring method that we propose here is based on mutual information scores of each candidate with the words in the context. We explore how far such a method can go when using the Web as a corpus. We estimate the counts by using the Waterloo MultiText System [Clarke and Terra 2003b] with a corpus of about one terabyte of text collected by a Web crawler.

\section{RELATED WORK}

The idea of using the Web as a corpus of texts has been exploited by many researchers. Radev and McKeown [1997] acquired different ways of referring to the 
same named entity, from the Web. Grefenstette [1999] used the Web for examplebased machine translation; Kilgarriff [2001] investigated the type of noise in Web data; Mihalcea and Moldovan [1999] and Agirre and Martinez [2000] used it as an additional resource for word sense disambiguation; Resnik [1999] mined the Web for bilingual texts; Turney [2001] used Web frequency counts to compute information retrieval-based mutual-information scores. In a Computational Linguistics special issue on the Web as a corpus [Kilgarriff and Grefenstette 2003], Keller and Lapata [2003] show that Web counts correlate well with counts collected from a balanced corpus: the size of the Web compensates for the noise in the data. In this paper we are using a very large corpus of Web pages to address a problem that has not been successfully solved before.

In fact, the only work that addresses exactly the same task is that of Edmonds [1997], as far as we are aware. Edmonds gives a solution based on a lexical cooccurrence network that included second-order co-occurrences. We use a much larger corpus and a simpler method, and we obtain much better results.

Our task has similarities to the word sense disambiguation task. Our nearsynonyms have senses that are very close to each other. In Senseval, some of the fine-grained senses are also close to each other, so they might occur in similar contexts, while the coarse-grained senses are expected to occur in distinct contexts. In our case, the near-synonyms are different words to choose from, not the same word with different senses.

Turney et al. [2003] addressed the multiple-choice synonym problem: given a word, choose a synonym for that word, among a set of possible solutions. In this case the solutions contain one synonym and some other (unrelated) words. They achieve high performance by combining classifiers. Clarke and Terra [2003a] addressed the same problem as Turney et al., using statistical associations measures computed with counts from the Waterloo terabyte corpus. In our case, all the possible solutions are synonyms of each other, and the task is to choose one that best matches the context: the sentence in which the original synonym is replaced with a gap. It is much harder to choose between words that are near-synonyms because the context features that differentiate a word from other words might be shared among the near-synonyms. Therefore the choice is done on the basis of a few features that are discriminant.

\section{A NEW STATISTICAL METHOD FOR NEAR-SYNONYM CHOICE}

Our method computes a score for each candidate near-synonym that could fill in the gap. The near-synonym with the highest score is the proposed solution. The score for each candidate reflects how well a near-synonym fits in with the context. It is based on the mutual information scores between a near-synonym and the content words in the context (we filter out the stopwords).

The pointwise mutual information $(P M I)$ between two words $x$ and $y$ compares the probability of observing the two words together (their joint probability) to the probabilities of observing $x$ and $y$ independently (the probability of occurring together by chance) [Church and Hanks 1991].

$$
P M I(x, y)=\log _{2} \frac{P(x, y)}{P(x) P(y)}
$$

ACM Transactions of Speech and Language Processing, Vol. 4, No. 1, 2007. 
The probabilities can be approximated by: $P(x)=C(x) / N, P(y)=C(y) / N$, $P(x, y)=C(x, y) / N$, where $C$ denote frequency counts and $N$ is the total number of words in the corpus. Therefore:

$$
P M I(x, y)=\log _{2} \frac{C(x, y) \cdot N}{C(x) \cdot C(y)}
$$

where $N$ can be ignored in comparisons, since is it the same in all the cases.

We model the context as a window of size $2 k$ around the gap (the missing word): $k$ words to the left and $k$ words to the right of the gap. If the sentence is $s=\cdots w_{1} \cdots w_{k}$ Gap $w_{k+1} \cdots w_{2 k} \cdots$, for each near-synonym $N S_{i}$ from the group of candidates, the score is computed by the following formula:

$$
\operatorname{Score}\left(N S_{i}, s\right)=\Sigma_{j=1}^{k} \operatorname{PMI}\left(N S_{i}, w_{j}\right)+\Sigma_{j=k+1}^{2 k} \operatorname{PMI}\left(N S_{i}, w_{j}\right) .
$$

We also experimented with the same formula when the sum is replaced with maximum to see whether a particular word in the context has higher influence than the sum of all contributions.

Because we are using the Waterloo terabyte corpus and we issue queries to its search engine, we have several possibilities of computing the frequency counts. $C(x, y)$ can be the number of co-occurrences of $x$ and $y$ when $y$ immediately follows $x$, or the distance between $x$ and $y$ can be up to $q$. We call $q$ the query frame size. The tool for accessing the corpus allows us to use various values for $q$ in queries. We used queries of the type $[q]>(x . . y)$, which asks how many times $x$ is followed by $y$ in a frame of size $q$.

The search engine also allows us to approximate words counts with document counts. If the counts $C(x), C(y)$, and $C(x, y)$ are approximated as the number of document in which they appear, we obtain the PMI-IR formula [Turney 2001]. The queries we need to send to the search engine are the same but they are restricted to document counts: $C(x)$ is the number of document in which $x$ occurs; $C(x, y)$ is the number of documents in which $x$ is followed by $y$ in a frame of size $q$; the query is formulated as $(\langle d o c\rangle . .\langle/ d o c\rangle)>[q]>(x . . y)$.

Other statistical association measures, such as log-likelihood, could be used. We tried only $P M I$ because it is easy to compute on a Web corpus and because [Clarke and Terra 2003a] showed that $P M I$ performed better than other measures in their experiments.

We present the results in Section 6.1, where we compare our method to a baseline algorithm that always chooses the most frequent near-synonyms and to Edmonds's method for the same task, on the same data set. First, however, we present two other methods to which we compare our results.

\section{THE ANTI-COLLOCATIONS METHOD}

For the task of near-synonym choice, another method that we implemented is the anti-collocations method. By anti-collocation we mean a combination of words that a native speaker would not use and therefore should not be used when automatically generating text. This method uses a knowledge-base of collocational behavior of near-synonyms that we acquired in previous work [Inkpen and Hirst 2002]. To build this knowledge-base, we acquired collocations of the near-synonyms, from a corpus. 


\begin{tabular}{l|l|}
\hline ghastly mistake & spelling mistake \\
*ghastly error & spelling error \\
ghastly blunder & $*$ spelling blunder \\
$*$ ghastly faux pas & *spelling faux pas \\
$*$ ghastly blooper & *spelling blooper \\
*ghastly solecism & *spelling solecism \\
$*$ ghastly goof & *spelling goof \\
$*$ ghastly contretemps & $*$ spelling contretemps \\
$*$ ghastly boner & $*$ spelling boner \\
$*$ ghastly slip & $*$ spelling slip
\end{tabular}

Table I. Examples of collocations and anti-collocations. The $*$ indicates the anti-collocations.

For each word that collocated with a near-synonym, we used a $t$-test (computed with Web counts collected through the AltaVista search engine) to learn whether the word forms a collocation or an anti-collocation with other near-synonyms in the same group. A fragment of the knowledge-base is presented in Table I, for the nearsynonyms of the word error and two collocate words ghastly and spelling. The lines marked by $*$ represent anti-collocations and the rest represent strong collocations.

The anti-collocations method simply ranks the strong collocations higher than the anti-collocations. In case of ties it chooses the most frequent near-synonym. In Section 6.2 we present the results of comparing this method to the method from the previous section.

\section{A SUPERVISED LEARNING METHOD}

We can also apply supervised learning techniques to our task. It is easy to obtain labelled training data, the same way we collected test data for the two unsupervised methods presented above. We train classifiers for each group of near-synonyms. The classes are the near-synonyms in the solution set. Each sentence is converted into a vector of features to be used for training the supervised classifiers. We used two types of features. One type of features are the scores of the left and right context with each class (i.e., with each near-synonym from the group). The number of features of this type is equal to twice the number of classes: one feature for the score between the near-synonym and the part of the sentence at the left of the gap, and one feature for the score between the near-synonym and the part of the sentence at the right of the gap. The second type of features are the words in the context windows. For each group of near-synonyms, we used as features the 500 mostfrequent words situated close to the gaps in a development set. The value of a word feature for each training example is 1 if the word is present in the sentence (at the left or at the right of the gap), and 0 otherwise. We trained classifiers using several machine learning algorithms, to see which one is best at discriminating among the near-synonyms. In Section 6.3, we present the results of several classifiers.

A disadvantage of the supervised method is that it requires training for each group of near-synonyms. Additional training would be required whenever we add more near-synonyms to our knowledge-base. An advantage of this method is that we could improve the accuracy by using a combination of classifiers and by trying other possible features. We think that part-of-speech features of the content words in the context may not be very useful since all the possible solutions have the same 


\begin{tabular}{|l|r|r|c|c|c|}
\cline { 3 - 6 } \multicolumn{2}{c|}{} & \multicolumn{4}{c|}{ Accuracy } \\
\hline $\begin{array}{l}\text { Test set } \\
\text { (Exp1 Data) }\end{array}$ & $\begin{array}{c}\text { Number } \\
\text { of cases }\end{array}$ & $\begin{array}{c}\text { Base- } \\
\text { line }\end{array}$ & $\begin{array}{c}\text { Edmonds's } \\
\text { method }\end{array}$ & $\begin{array}{c}\text { New } \\
\text { method } \\
\text { (Docs) }\end{array}$ & $\begin{array}{c}\text { New } \\
\text { method } \\
\text { (Words) }\end{array}$ \\
\hline difficult, hard, tough & 6,630 & $41.7 \%$ & $47.9 \%$ & $\mathbf{6 1 . 0} \%$ & $59.1 \%$ \\
\hline error, mistake, oversight & 1,052 & $30.9 \%$ & $48.9 \%$ & $\mathbf{6 6 . 4 \%}$ & $61.5 \%$ \\
\hline job, task, duty & 5,506 & $70.2 \%$ & $68.9 \%$ & $69.7 \%$ & $\mathbf{7 3 . 3} \%$ \\
\hline $\begin{array}{l}\text { responsibility, burden, } \\
\text { obligation, commitment }\end{array}$ & 3,115 & $38.0 \%$ & $45.3 \%$ & $64.1 \%$ & $\mathbf{6 6 . 0} \%$ \\
\hline material, stuff, substance & 1,715 & $59.5 \%$ & $64.6 \%$ & $68.6 \%$ & $\mathbf{7 2 . 2} \%$ \\
\hline give, provide, offer & 11,504 & $36.7 \%$ & $48.6 \%$ & $52.0 \%$ & $\mathbf{5 2 . 7} \%$ \\
\hline settle, resolve & 1,594 & $37.0 \%$ & $65.9 \%$ & $74.5 \%$ & $\mathbf{7 6 . 9 \%}$ \\
\hline \hline ALL (average) & 31,116 & $44.8 \%$ & $55.7 \%$ & $65.1 \%$ & $\mathbf{6 6 . 0 \%}$ \\
\hline
\end{tabular}

Table II. Comparison between the new statistical method from Section 3, baseline algorithm, and Edmonds's method. See details about Experiment 1 in Section 6.1.

part-of-speech and might have similar syntactic behavior. Maybe some function words immediately before the gaps could discriminate among the near-synonyms in some groups.

\section{EVALUATION}

\subsection{Comparison to Edmonds's method}

In this section we present results of the statistical method explained in Section 3. We compare our results with those of Edmonds [1997], whose solution used the texts from the year 1989 of the Wall Street Journal (WSJ) to build a lexical cooccurrence network for each of the seven groups of near-synonyms from Table II. The network included second-order co-occurrences. Edmonds used the WSJ 1987 texts for testing, and reported accuracies only a little higher than the baseline. The near-synonyms in the seven groups were chosen to have low polysemy. This means that some sentences with wrong senses of near-synonyms might be in the automatically produced test set, but hopefully not many.

For comparison purposes, in this section we use the same test data (WSJ 1987) and the same groups of near-synonyms. Our method is based on mutual information, not on co-occurrence counts. Our counts are collected from a much larger corpus. The seven groups of near-synonyms used by Edmonds are listed in the first column of Table II. If we would have used groups of synonyms from WordNet, we would probably obtain similar results, because the words in the seven groups differ only a little. Here are the words from the WordNet synsets:

mistake, error, fault

job, task, chore

duty, responsibility, obligation

difficult, hard

material, stuff

put up, provide, offer

decide, settle, resolve, adjudicate.

Before we look at the results, we mention that the accuracy values we compute are the percentage of correct choices when filling in the gap with the winning near-

ACM Transactions of Speech and Language Processing, Vol. 4, No. 1, 2007. 
synonym. The expected solution is the near-synonym that was originally in the sentence and it was taken out to create the gap. This measure is conservative, it does not consider cases when more than one solution is correct.

Table II presents the comparative results for seven groups of near-synonyms. The last row averages the accuracies for all the test sentences. The second column shows how many test sentences we collected for each near-synonym group. The third column is for the baseline algorithm that always chooses the most frequent near-synonym. The fourth column presents the results reported in [Edmonds 1997]. The fifth column presents the result of our method when using document counts in $P M I-I R$, and the last column is for the same method when using word counts in $P M I$. We show in bold the best accuracy figure for each data set. We notice that the automatic choice is more difficult for some near-synonym groups than for the others.

To fine-tune our statistical method, we used the data set for the near-synonyms of the word difficult collected from the WSJ 1989 corpus as a development set. We varied the context window size $k$ and the query frame $q$, and determined good values for the parameter $k$ and $q$. The best results were obtained for small window sizes, $k=1$ and $k=2$ (meaning $k$ words to the left and $k$ words to the right of the gap). For each $k$, we varied the query frame size $q$. The results are best for a relatively small query frame, $q=3,4,5$, when the query frame is the same or slightly larger then the context window. The results are worse for a very small query frame, $q=1,2$. The results presented in the rest of the paper are for $k=2$ and $q=5$. For all the other data sets used in this paper (from WSJ 1987 and BNC) we use the parameter values as determined on the development set.

Table II shows that the performance is generally better for word counts than for document counts. Therefore, we prefer the method that uses word counts (which is also faster in our particular setting). The difference between them is not statistically significant. Our statistical method performs significantly better than Edmond's method and than the baseline algorithm. For all the results presented in this paper, statistical significance tests were done using the paired $t$-test, as described in [Manning and Schütze 1999], page 209.

In summary, the results are better for smaller context windows, for the sum of all the $P M I$ s with the words in the context window, not for taking the maximum contribution. The performance decreases with larger query frames $q=5,6, \ldots, 20$ and degrades sharply when $q$ is unlimited (the words are in the same document no matter at what distance). Error analysis reveals that more often incorrect choices happen when the context is weak: very short sentences, or sentences with very few content words.

On average, our method performs 22 percentage points better than the baseline algorithm, and 10 percentage points better than Edmonds's method. Its performance is similar to that of the supervised method (see Section 6.3). An important advantage of our method is that it works on any group of near-synonyms without training, whereas Edmonds's method required a lexical co-occurrence network to be built in advance for each group of near-synonyms and the supervised method required training for each near-synonym group. 
(1) benefit, advantage, favor, gain, profit

(2) low, gush, pour, run, spout, spurt, squirt, stream

(3) deficient, inadequate, poor, unsatisfactory

(4) afraid, aghast, alarmed, anxious, apprehensive, fearful, frightened, scared, terrorstricken

(5) disapproval, animadversion, aspersion, blame, criticism, reprehension

(6) mistake, blooper, blunder, boner, contretemps, error, faux pas, goof, slip, solecism

(7) alcoholic, boozer, drunk, drunkard, lush, sot

(8) leave, abandon, desert, forsake

(9) opponent, adversary, antagonist, competitor, enemy, foe, rival

(10) thin, lean, scrawny, skinny, slender, slim, spare, svelte, willowy, wiry

(11) lie, falsehood, fib, prevarication, rationalization, untruth

Fig. 2. Near-synonyms used in the evaluation experiments in Section 6.2.

\subsection{Comparison to the anti-collocations method}

In a second experiment we compare the results of our methods with the anticollocations method described in Section 4. We use the data set from [Inkpen and Hirst 2002], which contains sentences from the first half of the British National Corpus, with near-synonyms from the eleven groups listed in Figure 2. The number of near-synonyms in each group is higher compared with WordNet synonyms, because they are taken from [Hayakawa 1994], a dictionary that explains differences between near-synonyms. Moreover we retain only the sentences in which at least one of the context words is in our previously acquired knowledge-base of nearsynonym collocations. That is, the anti-collocations method works only if we know how a word in the context collocates with the near-synonyms from a group. For the sentences that do not contain collocations or anti-collocations, it will perform no better than the baseline, because the information needed by the method is not available in the knowledge-base. Even if we increase the coverage of the knowledgebase, the anti-collocations method might still fail too often due to words that were not included.

Table III presents the results of the comparison. We used two data sets: TestSample, which includes at most two sentences per collocation (the first two sentences from the corpus); and TestAll, which includes all the sentences with collocations as they occurred in the corpus. The reason to do these two tests was to not bias the results due to frequent collocations.

The last two columns are the accuracies achieved by our new method. The second last column shows the results of the new method when the word counts are approximated with document counts. The improvement over the baseline is 16 to 27 percentage points. The improvement over the anti-collocations method is 10 to 17 percentage points.

\subsection{Comparison to supervised learning}

We present the results of the supervised method from Section 5 on the data sets used in Section 6.1. As explained before, the data sets contains sentences with a lexical gap. For each of the seven groups of near-synonyms, the class to choose 


\begin{tabular}{|l|c|c|c|c|c|}
\cline { 3 - 6 } \multicolumn{2}{c|}{} & \multicolumn{4}{c|}{ Accuracy } \\
\hline $\begin{array}{l}\text { Test set } \\
\text { (Exp2 Data) }\end{array}$ & $\begin{array}{c}\text { Number } \\
\text { of } \\
\text { cases }\end{array}$ & Baseline & $\begin{array}{c}\text { Anti-collocations } \\
\text { method }\end{array}$ & $\begin{array}{c}\text { New } \\
\text { method } \\
\text { (Docs) }\end{array}$ & $\begin{array}{c}\text { New } \\
\text { method } \\
\text { Words) }\end{array}$ \\
\hline TestSample & 171 & $57.0 \%$ & $63.3 \%$ & $\mathbf{7 5 . 6 \%}$ & $73.3 \%$ \\
\hline TestAll & 332 & $48.5 \%$ & $58.6 \%$ & $70.0 \%$ & $\mathbf{7 5 . 6 \%}$ \\
\hline
\end{tabular}

Table III. Comparison between the new statistical method from Section 3 and the anti-collocations method from Section 4. See details about Experiment 2 in Section 6.2.

\begin{tabular}{|l|c|c|}
\hline ML method (Weka) & Features & Accuracy (averaged) \\
\hline Decision Trees & PMI scores & $65.4 \%$ \\
\hline Decision Rules & PMI scores & $65.5 \%$ \\
\hline Naive Bayes & PMI scores & $52.5 \%$ \\
\hline K-Nearest Neighbor & PMI scores & $64.5 \%$ \\
\hline Kernel Density & PMI scores & $60.5 \%$ \\
\hline Boosting (Decision Stumps) & PMI scores & $\mathbf{6 7 . 7} \%$ \\
\hline Naive Bayes & 500 word features & $\mathbf{6 8 . 0} \%$ \\
\hline Decision Trees & 500 word features & $67.0 \%$ \\
\hline Naive Bayes & PMI + 500 word features & $66.5 \%$ \\
\hline Boosting (Decision Stumps) & PMI + 500 word features & $\mathbf{6 9 . 2} \%$ \\
\hline
\end{tabular}

Table IV. Comparative results for the supervised learning method using various ML learning algorithms (Weka), averaged over the seven groups of near-synonyms from the Experiment 1 data set.

\begin{tabular}{|l|r|r|r|c|c|}
\cline { 3 - 6 } \multicolumn{2}{c|}{} & \multicolumn{5}{c|}{ Accuracy } \\
\hline Test set & $\begin{array}{c}\text { Number } \\
\text { of cases }\end{array}$ & $\begin{array}{c}\text { Base- } \\
\text { line }\end{array}$ & $\begin{array}{c}\text { Supervised } \\
\text { Boosting } \\
(\text { PMI })\end{array}$ & $\begin{array}{c}\text { Supervised } \\
\text { Boosting } \\
\text { (PMI+words) }\end{array}$ & $\begin{array}{c}\text { Unsuper- } \\
\text { vised } \\
\text { method }\end{array}$ \\
\hline difficult, hard, tough & 6,630 & $41.7 \%$ & $55.8 \%$ & $57.3 \%$ & $\mathbf{5 9 . 1} \%$ \\
\hline error, mistake, oversight & 1,052 & $30.9 \%$ & $68.1 \%$ & $\mathbf{7 0 . 8} \%$ & $61.5 \%$ \\
\hline job, task, duty & 5,506 & $70.2 \%$ & $86.5 \%$ & $\mathbf{8 6 . 7} \%$ & $73.3 \%$ \\
\hline $\begin{array}{l}\text { responsibility, burden, } \\
\text { obligation, commitment }\end{array}$ & 3,115 & $38.0 \%$ & $66.5 \%$ & $\mathbf{6 6 . 7} \%$ & $66.0 \%$ \\
\hline material, stuff, substance & 1,715 & $59.5 \%$ & $70.4 \%$ & $71.0 \%$ & $\mathbf{7 2 . 2} \%$ \\
\hline give, provide, offer & 11,504 & $36.7 \%$ & $53.0 \%$ & $\mathbf{5 6 . 1} \%$ & $52.7 \%$ \\
\hline settle, resolve & 1,594 & $37.0 \%$ & $74.0 \%$ & $75.8 \%$ & $\mathbf{7 6 . 9} \%$ \\
\hline \hline ALL (average) & 31,116 & $44.8 \%$ & $67.7 \%$ & $\mathbf{6 9 . 2} \%$ & $66.0 \%$ \\
\hline
\end{tabular}

Table V. Comparison between the unsupervised statistical method from Section 3 and the supervised method described in Section 5, on the Experiment 1 data sets. The results of two of the best supervised classifiers are presented.

from in order to fill in the gaps is one of the near-synonyms in each cluster. We implemented classifiers that use as features either the PMI scores of the left and right context with each class, or the words in the context windows, or both types of features combined. We used as features the 500 most-frequent words for each group of near-synonyms. We report accuracies for 10-fold cross-validation. We used the Weka collection of machine learning algorithms [Witten and Frank 2000].

Table IV presents the results, averaged for the seven groups of near-synonyms, of several classifiers from the Weka package. The classifiers that use PMI features are 
Decision Trees, Decision Rules, Naive Bayes, K-Nearest Neighbor, Kernel Density, and Boosting a weak classifier (Decision Stumps - which are shallow decision trees). Then a Naive Bayes classifier that uses only the word features is presented, and the same type of classifiers with both types of features. The other classifiers from the Weka package were also tried, but the results did not improve and these algorithms had difficulties in scaling up. In particular, when using the 500 word features for each training example, only the Naive Bayes algorithm was able to run in reasonable time. We noticed that the Naive Bayes classifier performs very poorly on PMI features only ( $55 \%$ average accuracy), but performs very well on word features (68\% average accuracy). In contrast, the Decision Tree classifier performs well on PMI features, especially when using boosting with Decision Stumps. When using both the PMI scores and the word features, the results are slightly higher. It seems that both types of features are sufficient for training a good classifier, but combining them adds value.

Table V presents the detailed results of two of the supervised classifiers, and repeats, for easier comparison, the results of the unsupervised statistical method from Section 6.1. The supervised classifier that uses only PMI scores performs similar the unsupervised method. The best supervised classifier, that uses both types of features, performs slightly better than the unsupervised statistical method, but the difference is not statistically significant. We conclude that the results of the supervised methods and the unsupervised statistical method are similar. An important advantage of our unsupervised method is that it works on any group of near-synonyms without training.

\subsection{Comparison to language models}

Since one of the main applications of our methods is lexical choice in machine translation and statistical NLG, we need to compare our methods with the current mainstream method for lexical choice: language modeling. The sum of mutual information scores bears some similarity to a bigram language model. For example $P M I\left(w_{1}, w_{2}\right)+P M I\left(w_{2}, w_{3}\right)$ can be rewritten as:

$$
\begin{gathered}
P M I\left(w_{1}, w_{2}\right)+P M I\left(w_{2}, w_{3}\right)=\log _{2} \frac{P\left(w_{1}, w_{2}\right)}{P\left(w_{1}\right) P\left(w_{2}\right)}+\log _{2} \frac{P\left(w_{2}, w_{3}\right)}{P\left(w_{2}\right) P\left(w_{3}\right)}= \\
=\frac{\log _{2} P\left(w_{1}, w_{2}\right) P\left(w_{2}, w_{3}\right)}{P\left(w_{1}\right) P\left(w_{2}\right)^{2} P\left(w_{3}\right)}=\frac{\log _{2} P\left(w_{2} \mid w_{1}\right) P\left(w_{3} \mid w_{2}\right)}{P\left(w_{2}\right)^{2} P\left(w_{3}\right)^{2}}
\end{gathered}
$$

Lexical choice in most statistical Machine Translation (MT) systems today is heavily determined by the language model, and it has proven very difficult in practice to apply state-of-the-art Word Sense Disambiguation / lexical choice models to obtain improvements over a baseline statistical MT with a language model [Carpuat and $\mathrm{Wu} 2005]$.

We do not go into details here, but in previous work [Inkpen and Hirst 2006] we compared the results of the anti-collocations method to the results of using a 3 -gram language model. This language model is part on the HALogen NLG system [Langkilde and Knight 1998] and it was built on 250 million words newspaper text: AP Newswire, year 1988 and 1990; SJM, year 1991; and WSJ, years 1987-1988 and 


\begin{tabular}{|l|c|c|c|c|}
\hline Test set & $\begin{array}{c}\text { J1-J2 } \\
\text { Agreement }\end{array}$ & $\begin{array}{c}\text { J1 } \\
\text { Accuracy }\end{array}$ & $\begin{array}{c}\text { J2 } \\
\text { Accuracy }\end{array}$ & $\begin{array}{c}\text { System } \\
\text { Accuracy }\end{array}$ \\
\hline difficult, hard, tough & $72 \%$ & $70 \%$ & $76 \%$ & $53 \%$ \\
\hline error, mistake, oversight & $82 \%$ & $84 \%$ & $84 \%$ & $68 \%$ \\
\hline job, task, duty & $86 \%$ & $92 \%$ & $92 \%$ & $78 \%$ \\
\hline $\begin{array}{l}\text { responsibility, burden, } \\
\text { obligation, commitment }\end{array}$ & $76 \%$ & $82 \%$ & $76 \%$ & $66 \%$ \\
\hline material, stuff, substance & $76 \%$ & $82 \%$ & $74 \%$ & $64 \%$ \\
\hline give, provide, offer & $78 \%$ & $68 \%$ & $70 \%$ & $52 \%$ \\
\hline settle, resolve & $80 \%$ & $80 \%$ & $90 \%$ & $77 \%$ \\
\hline \hline ALL (average) & $78.5 \%$ & $79.7 \%$ & $80.2 \%$ & $65.4 \%$ \\
\hline
\end{tabular}

Table VI. Experiments with two human judges on a random subset of the Experiment 1 data set.

1990-1994. The comparison of the two methods was done on different groups of near-synonyms, including some of the groups from the Experiment 1 data set. The anti-collocation method performed better on the task of choosing the best nearsynonym in a context, therefore so would the statistical method based on PMI (because we showed in Section 6.2 that our PMI-based method achieves higher accuracy than the anti-collocations method).

\subsection{Experiments with human judges}

We asked two human judges, native speakers of English, to guess the missing word in a random sample of the Experiment 1 data set (50 sentences for each of the 7 groups of near-synonyms, 350 sentences in total). The results in Table VI show that the agreement between the two judges is high $(78.5 \%)$, but not perfect. This means the task is difficult, even if some wrong senses in the automatically-produced test data might have made the task easier in a few cases.

The human judges were allowed to choose more than one correct answer when they were convinced that more than one near-synonym fits well in the context. They used this option sparingly, only in $5 \%$ of the 350 sentences. In future work, we plan to allow the system to make more than one choice when appropriate (for example when the second choice has very close score to the first choice).

Taking the accuracy achieved by the human judges as upper limit, we conclude that the automatic method has room for improvement of approximately 10-15 percentage points.

\section{APPLICATIONS}

\subsection{Intelligent thesaurus}

The intelligent thesaurus that we are developing is an interactive application that presents the user with a list of alternative near-synonyms, and, unlike standard thesauri, it orders the choices to match the writing context and explains the differences between the possible choices.

The new statistical method presented in this paper (in Section 3) allows us to order the near-synonyms according to how well they fit into the writing context. When composing a text, if the writer is unhappy with a word, he/she can select that word and ask for a better substitute. The intelligent thesaurus provides alternative 


\begin{tabular}{|l|c|c|}
\hline Test set & $\begin{array}{c}\text { Accuracy for } \\
\text { the first choice }\end{array}$ & $\begin{array}{c}\text { Accuracy for } \\
\text { the first two choices }\end{array}$ \\
\hline Experiment 1 Data, ALL & $66.0 \%$ & $\mathbf{8 8 . 5 \%}$ \\
\hline Experiment 2 Data, TestSample & $73.3 \%$ & $\mathbf{9 4 . 1 \%}$ \\
\hline Experiment 2 Data, TestAll & $75.6 \%$ & $\mathbf{8 7 . 5 \%}$ \\
\hline
\end{tabular}

Table VII. Accuracies for the first two choices as ordered by an interactive intelligent thesaurus.

\begin{tabular}{|l|c|c|}
\hline Test set & $\begin{array}{c}\text { Accuracy for } \\
\text { the first choice }\end{array}$ & $\begin{array}{c}\text { Accuracy for } \\
\text { the first two choices }\end{array}$ \\
\hline Experiment 1 Data, ALL & $58.05 \%$ & $\mathbf{8 4 . 8 2 \%}$ \\
\hline Experiment 2 Data, TestSample & $57.4 \%$ & $\mathbf{7 5 . 1 \%}$ \\
\hline Experiment 2 Data, TestAll & $56.1 \%$ & $\mathbf{7 7 . 4 \%}$ \\
\hline
\end{tabular}

Table VIII. Results of the statistical method when only the left context is considered, for the data sets from Section 6.1.

near-synonyms, in a context-dependent manner. Our experiments show that the accuracy of the first choice being the best choice is 66 to $75 \%$; therefore there will be cases when the writer will not choose the first alternative. But the accuracy for first two choices is quite high, around $90 \%$, as presented in Table VII.

If the writer is in the process of writing and selects the last word to be replaced with a near-synonym proposed by the thesaurus, then only the context on the left of the word can be used for ordering the alternatives. Our method can be easily adapted to consider only the context on the left of the gap. The results of this case are presented in Table VIII, for the data sets used in the previous sections. The accuracy values are lower than in the case when both the left and the right context are considered (Table VII). This is due in part to the fact that some sentences in the test sets have very little left context, or no left context at all. On another hand, many times the writer composes a sentence or paragraph and then she/he goes back to change a word that does not sound right. In this case, both the left and right context will be available.

In the intelligent thesaurus, we could combine the supervised and unsupervised method, by using a supervised classifier when the confidence in the classification is high, and by using the unsupervised method otherwise. Also the unsupervised statistical method would be used for the groups of near-synonyms for which a supervised classifier was not previously trained.

Figure 3 presents a screen short of the current implementation of the intelligent thesaurus application. The system allows the user to specify which synonym inventory to use. We integrated Roget's thesaurus [Roget 1852] in order to increase coverage (the number of words for which alternatives are available). We used the interface provided by Jarmasz and Szpakowicz [2001]. Roget's thesaurus contains among the related word several phrases (as seen in Figure 3). In future work, we need to find new ways to score these phrases, because the PMI formula that we used gives them too high scores.

The system also allows to specify from which corpus to collect the counts (including from the Web, through the Google and Yahoo API). The system performs 


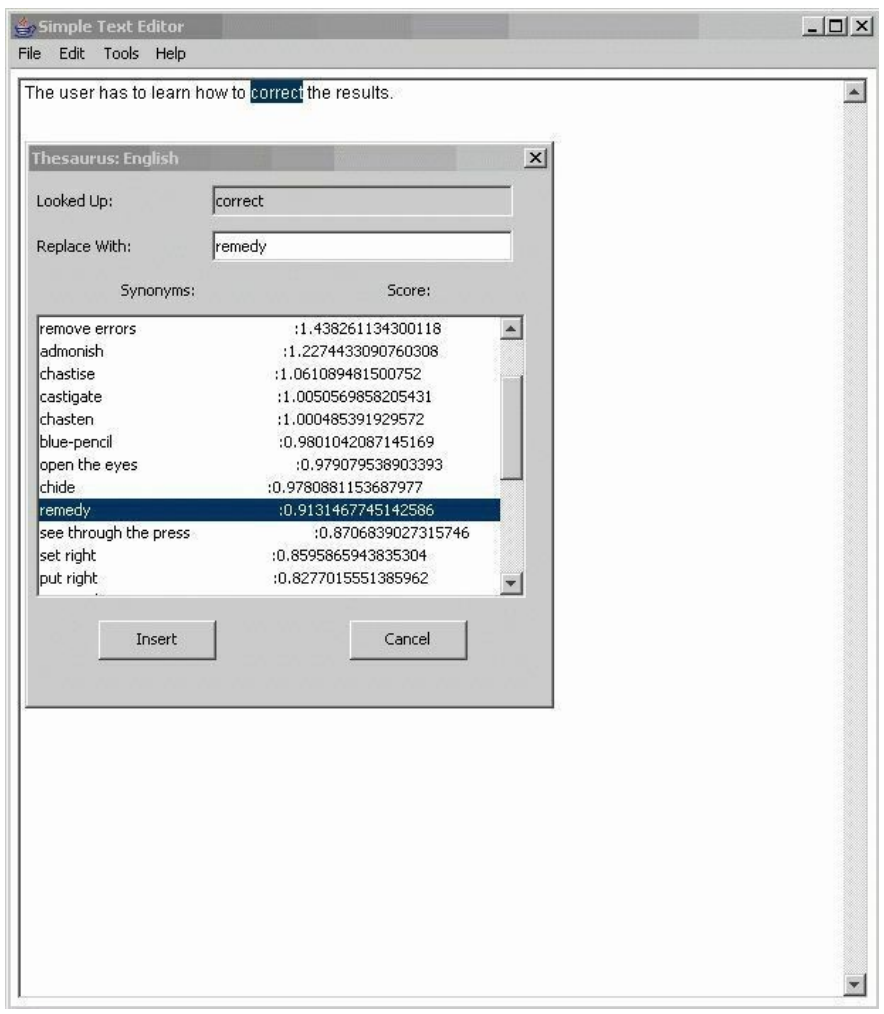

Fig. 3. Screen shot of the intelligent thesaurus application.

part-of-speech tagging (using $\mathrm{QTAG}^{1}$ ) on the text being written in the text editor, in order to be able to suggest only synonyms with the right part-of-speech.

Our intelligent thesaurus can present in a separate window explanations of how the near-synonyms differ in the lexical nuances they carry. This kind of knowledge is available in a limited form, from our previous work [Inkpen and Hirst 2001] on extracting information from a special dictionary of synonym discrimination [Hayakawa 1994]. The coverage is limited in the sense that this knowledge-base contains only 5452 words, in 909 groups of near-synonyms. The explanations include denotational nuances (what do the near-synonyms imply or suggest), how formal or informal they are, and how positive or negative they might sound; also examples of usage are provided. We do not expand on this aspect of our intelligent thesaurus here because the focus of this paper is on the collocational properties of the near-synonyms.

\subsection{Natural language generation}

In previous work [Inkpen and Hirst 2006] we presented and evaluated an NLG system that pays particular attention to choosing the right near-synonyms. This system extended HALogen[Langkilde and Knight 1998] with two extra modules: a

\footnotetext{
${ }^{1}$ http://www.english.bham.ac.uk/staff/omason/software/qtag.html
} 
symbolic and a statistical module for near-synonym choice.

Without going into details, we mention that the symbolic module deals with nuances of meaning in an explicit way. Connotations or implications of certain concepts are represented using an interlingual representation language and concepts from an ontology. The user can specify what nuances of meaning are preferred in the generated sentence, in addition to the main meaning. Alternatively, the preferences could come from the sentence in the source language, if our NLG system is used as part of an interlingual machine translation system. The system knows which near-synonyms carry which nuances of meaning because it contains the knowledge automatically acquired from the special dictionary of synonym discrimination [Hayakawa 1994].

The statistical module uses the anti-collocations method to choose near-synonyms that collocate well with the other words in the context. We plan to replace it with the PMI-based method, in order to increase coverage and accuracy.

\section{CONCLUSION}

We presented a statistical method of choosing the best near-synonym in a context. We compared this method with two previous methods (Edmonds's method and the anti-collocations method) and showed that the performance improves considerably. We also show that our unsupervised statistical method performs comparably to a supervised learning method.

Our method based on PMI scores performs well, despite the well-known limitations of PMI when used with corpora. PMI tends to have problems mostly on very small counts, but it works reasonably with larger counts. Our web corpus is quite large, therefore the problem of small counts does not appear.

We combine symbolic and statistical knowledge in two applications: an intelligent thesaurus; and a natural language generation system that has knowledge of nuances of meaning of near-synonyms.

The accuracy of 66 to $75 \%$ is not enough for automatic choice, but helps complement the choices made by using symbolic knowledge. In the intelligent thesaurus, we do not make the near-synonym choice automatically, but we let the user choose. The first choice offered by the thesaurus is the best one quite often, and if we consider the first two choices, they are correct $90 \%$ of the time.

In this paper we focused on idiomatic usage of near-synonyms, while in previous work we looked at nuances of meaning and differences between near-synonyms in terms of connotations and implications [Inkpen and Hirst 2006]. There are, though, some implications that are captured by idiomatic usage. For example, Church and Hanks [1991] presented associations (collocations, but not necessarily between adjacent wors) for the near-synonyms ship and boat; they suggest that a lexicographer looking at these associations can infer that boats are generally smaller than ships, because they are found in rivers and lakes and are used for small jobs (e.g., fishing, police, pleasure), whereas ships are found in seas and are used for serious business (e.g., cargo, war).

In future work, we plan to investigate the possibility to automatically infer this kind of knowledge or to validate already acquired knowledge. Words that do not associate with a near-synonym but associate with all the other near-synonyms in a

ACM Transactions of Speech and Language Processing, Vol. 4, No. 1, 2007. 
cluster could tell us something about its nuances of meaning. For example terrible slip is an anti-association, whereas terrible associates with mistake, blunder, error. This is an indication that slip is a minor error. By further generalization, the associations could become conceptual associations. This may allow the automatic learning of denotational distinctions between near-synonyms from free text. The concepts that are common to all the near-synonyms in a cluster could be part of their main meaning, while those that associate only with one near-synonym could be part of their implied nuances of meaning.

Future work includes a near-synonym sense disambiguation module to ensure that the intelligent thesaurus does not offer alternatives for wrong senses of words. In addition to the groups of synonyms from WordNet, Roget, and dictionaries of synonyms, we could acquire synonyms from corpora, so that the intelligent thesaurus can offer alternatives for a very large number of words. There is research done on acquiring distributionally similar words [Lin 1998], but they include, in addition to near-synonyms, words that are in other relations. Lin et al. [2003] looked at filtering out the antonyms, using specific co-occurrence patterns. Words that are in other relations could also be filtered out. One way to do this could be to collect signatures for each potential near-synonym - words that associate with it in many contexts. For two candidate words, if one signature is contained in the other, the words are probably in a IS-A relation; if the signatures overlap totally, it is a true near-synonymy relation; if the signatures overlap partially, it is a different kind of relation.

\section{Acknowledgments}

We are most grateful to Egidio Terra, Charlie Clarke, and the School of Computer Science of the University of Waterloo, for allowing us to use their terabyte webpage corpus and the MultiText system. We thank Peter Turney and his colleagues at IIT/NRC Ottawa for giving us access to their local copy of the corpus. We also thank Peter Turney for sharing his Perl code for remote access to the corpus, and for his comments on the draft of this paper. We thank Graeme Hirst for comments on earlier versions of this paper. We thank Jayakumar Balasingham and Apoorve Chokshi for their contribution to the implementation of the Intelligent Thesaurus application and Mario Jarmasz for allowing us to use his software interface to Roget's thesaurus. Our work is funded by the Natural Sciences and Engineering Research Council of Canada and the University of Ottawa.

\section{REFERENCES}

AgirRe, E. And Martinez, D. 2000. Exploring automatic word sense disambiguation with decision lists and the Web. In Proceedings of the Workshop on Semantic Annotation And Intelligent Content, COLING 2000. Saarbrücken/Luxembourg/Nancy.

CARpuat, M. AND WU, D. 2005. Evaluating the word sense disambiguation performance of statistical machine translation. In Proceedings of the 43th Annual Meeting of the Association for Computational Linguistics. Ann Arbor, Michigan, USA, 120-125.

Church, K., Gale, W., Hanks, P., And Hindle, D. 1991. Using statistics in lexical analysis. In Lexical Acquisition: Using On-line Resources to Build a Lexicon, U. Zernik, Ed. Lawrence Erlbaum, 115-164.

Church, K. And Hanks, P. 1991. Word association norms, mutual information and lexicography. Computational Linguistics 16 (1), 22-29. 
Clarke, C. L. A. and Terra, E. 2003a. Frequency estimates for statistical word similarity measures. In Proceedings of the Human Language Technology Conference of the North American Chapter of the Association for Computational Linguistics (HLT-NAACL 2003). Edmonton, Canada, 165-172.

Clarke, C. L. A. and Terra, E. 2003b. Passage retrieval vs. document retrieval for factoid question answering. In Proceedings of the 26th Annual International ACM SIGIR Conference on Research and Development in Information Retrieval. Toronto, Canada, 427-428.

EDmonds, P. 1997. Choosing the word most typical in context using a lexical co-occurrence network. In Proceedings of the 35th Annual Meeting of the Association for Computational Linguistics. Madrid, Spain, 507-509.

Grefenstette, G. 1999. The World Wide Web as a resource for example-based machine translation tasks. In Proceedings of the ASLIB Conference on Translating and Computers. London, UK.

Hayakawa, S. I., Ed. 1994. Choose the Right Word. Second Edition, revised by Eugene Ehrlich. HarperCollins Publishers.

InKPEN, D. Z. AND HiRst, G. 2001. Building a lexical knowledge-base of near-synonym differences. In Proceedings of the Workshop on WordNet and Other Lexical Resources, Second Meeting of the North American Chapter of the Association for Computational Linguistics (NAACL 2001). Pittsburgh, USA, 47-52.

InkPen, D. Z. AND Hirst, G. 2002. Acquiring collocations for lexical choice between nearsynonyms. In Proceedings of the Workshop on Unsupervised Lexical Acquisition, 40th Annual Meeting of the Association for Computational Linguistics (ACL 2002). Philadelphia, USA, $67-76$.

InKPEN, D. Z. AND HiRst, G. 2003. Near-synonym choice in natural language generation. In Proceedings of the International Conference RANLP-2003 (Recent Advances in Natural Language Processing). Borovets, Bulgaria, 204-211.

InKPen, D. Z. And Hirst, G. 2006. Building and using a lexical knowledge-base of near-synonym differences. Computational Linguistics 32 (2).

Jarmasz, M. And SzPakowicz, S. 2001. The design and implementation of an electronic lexical knowledge base. In Proceeding of the 14th Biennial Conference of the Canadian Society for Computational Studies of Intelligence (AI 2001). Ottawa, Canada, 325-334.

Keller, F. and Lapata, M. 2003. Using the Web to obtain frequencies for unseen bigrams. Computational Linguistics 29 (3), 459-484.

Kilgarriff, A. 2001. Web as corpus. In Proceedings of the 2001 Corpus Linguistics conference. Lancaster, UK, 342-345.

Kilgarriff, A. and Grefenstette, G. 2003. Introduction to the special issue on the Web as a corpus. Computational Linguistics 29 (3), 333-347.

LangKilde, I. And Knight, K. 1998. The practical value of N-grams in generation. In Proceedings of the 9th International Natural Language Generation Workshop. Niagara-on-the-Lake, Canada, 248-255.

LIN, D. 1998. Automatic retrieval and clustering of similar words. In Proceedings of the 36th Annual Meeting of the Association for Computational Linguistics joint with 17th International Conference on Computational Linguistics (ACL-COLING'98). Montreal, Quebec, Canada, 768-774.

Lin, D., ZhaO, S., Qin, L., AND Zhou, M. 2003. Identifying synonyms among distributionally similar words. In Proceedings of the Eighteenth Joint International Conference on Artificial Intelligence (IJCAI-03). Acapulco, Mexico.

Manning, C. And Schütze, H. 1999. Foundations of Statistical Natural Language Processing. The MIT Press, Cambridge, Massachusetts.

Minalcea, R. And Moldovan, D. 1999. A method for word sense disambiguation from unrestricted text. In Proceedings of the 37th Annual Meeting of the Association for Computational Linguistics. Maryland, USA, 152-158.

ACM Transactions of Speech and Language Processing, Vol. 4, No. 1, 2007. 
RADEv, D. AND MCKeown, K. R. 1997. Building a generation knowledge source using internetaccessible newswire. In Proceedings of the Fifth ACL Conference on Applied Natural Language Processing ANLP'97. Washington, DC, 221-228.

Resnik, P. 1999. Mining the Web for bilingual text. In Proceedings of the 37th Annual Meeting of the Association for Computational Linguistics. Maryland, USA, 527-534.

Roget, P. M., Ed. 1852. Roget's Thesaurus of English Words and Phrases. Longman Group Ltd., Harlow, Essex, England.

Turney, P. 2001. Mining the Web for synonyms: PMI-IR versus LSA on TOEFL. In Proceedings of the Twelfth European Conference on Machine Learning (ECML 2001). Freiburg, Germany, 491-502.

Turney, P., Littman, M., Bigham, J., And Shnayder, V. 2003. Combining independent modules to solve multiple-choice synonym and analogy problems. In Proceedings of the International Conference RANLP-2003 (Recent Advances in Natural Language Processing). Borovets, Bulgaria, 482-489.

Witten, I. H. AND Frank, E. 2000. Data Mining: Practical machine learning tools with Java implementations. Morgan Kaufmann, San Francisco, USA. 